\title{
Enhanced Thermal Shock Resistance of Ceramics through Biomimetically Inspired Nanofins
}

\author{
Fan Song, ${ }^{1,2, *}$ Songhe Meng, ${ }^{3}$ Xianghong Xu, ${ }^{1}$ and Yingfeng Shao ${ }^{1}$ \\ ${ }^{1}$ State Key Laboratory of Nonlinear Mechanics (LNM), Institute of Mechanics, Chinese Academy of Sciences, Beijing 100080, \\ People's Republic of China \\ ${ }^{2}$ Center for Biomechanics and Bioengineering (CBB), Institute of Mechanics, Chinese Academy of Sciences, Beijing 100080, \\ People's Republic of China \\ ${ }^{3}$ Center for Composite Materials, Harbin Institute of Technology, Harbin 150080, People's Republic of China
}

(Received 14 July 2009; published 22 March 2010)

\begin{abstract}
We propose here a new method to make ceramics insensitive to thermal shock up to their melting temperature. In this method the surface of ceramics was biomimetically roughened into nanofinned surface that creates a thin air layer enveloping the surface of the ceramics during quenching. This air layer increases the heat transfer resistance of the surface of the ceramics by about 10000 times so that the strong thermal gradient and stresses produced by the steep temperature difference in thermal shock did not occur both on the actual surface and in the interior of the ceramics. This method effectively extends the applications of existing ceramics in the extreme thermal environments.
\end{abstract}

DOI: 10.1103/PhysRevLett.104.125502

PACS numbers: 62.25.Mn, 44.10.+i, 65.90.+i, 81.40.Np

Ceramics are renowned for their excellent resistances to the extreme environments including high temperature and heavy chemical corrosion, but their poor resistance to thermal shock has been a major challenge in the thermal engineering for a long time [1-3]. Thermal shock, which catastrophically decreases the ceramic strength at a very low temperature in comparison with their melting points, often occurs in the thermostructural engineering in the engines with ceramic components and in routine daily activities such as cooling a boiling egg in iced water [412]. $\mathrm{ZrB}_{2}$ plus 30 vol. \% $\mathrm{SiC}$, for example, which is a refractory ceramic with a high melting point of about $3245^{\circ} \mathrm{C}$ and is currently expected to be applied to the airframe leading edges and noses on sharp-bodied reentry vehicles, exhibits a high mean strength of about $830 \mathrm{MPa}$, the temperature of thermal shock is about $395^{\circ} \mathrm{C}$ $[3,13,14]$. However, above this temperature value, the strength suddenly decreases to about $120 \mathrm{MPa}$; i.e., its residual strength is less than $15 \%$ of the intrinsic strength when quenched from a temperature of $12 \%$ of its melting point $[3,13]$. Although the primary failure mechanism for ceramics in thermal shock is considered to be crack initiation, when the stresses imposed by a thermal gradient exceed the strength of the materials [4-6], the possible mechanisms yielding the catastrophic reduction of the strength have not been well understood yet [8-13]. In particular, an effective method overcoming the failure of ceramics in thermal shock has not been proposed up to now. In this study, we present a generic method that makes ceramic materials insensitive to thermal shock up to their melting points and discuss briefly a possible physical origin of this method.

The ceramic studied here is $\mathrm{ZrB}_{2}-20 \% \mathrm{SiC}_{p}-5 \%$ AlN (ZSA), a refractory ceramic fabricated by hotpressing
[15]. Its flexural strength at room temperature is $738.6 \pm$ 45.4 $\mathrm{MPa}$ and the melting point in common atmospheric pressure about $3210^{\circ} \mathrm{C}$. We used the method of rapidly quenching to investigate the properties of the ceramic during thermal shock [2]. Specimens were first cut into $3 \times$ $4 \times 36 \mathrm{~mm}$ bars at the ambient temperature of about $20^{\circ} \mathrm{C}$, and then heated with a rate of $10{ }^{\circ} \mathrm{C} / \mathrm{min}$ up to a preset temperature and held at this temperature for $20 \mathrm{~min}$. After that, the heated specimens were placed into water at the ambient temperature for quenching and maintained for $10 \mathrm{~min}$. The strength was then measured using three-point bending at the ambient temperature, and at each of the preset temperature points, five specimens were tested. The experimental results indicate that the quenched specimens exhibit a mean strength identical to that of an unquenched one as long as the temperature is smaller than $400{ }^{\circ} \mathrm{C}$. But above this value, the strength suddenly decreased to 105.4 \pm 47.2 MPa, which was less than $15 \%$ of their intrinsic strength, as shown in Fig. 1(a). Our experimental observations proved that there are cracks produced on the surfaces of the ceramics when the quenching temperature was greater than $400{ }^{\circ} \mathrm{C}$, and the higher the temperature is, the more the number of the surface crack is, as shown in Fig. 1(b). Moreover, when the quenching temperature is greater than $800^{\circ} \mathrm{C}$, the surfaces of the ceramics appeared to a very thin oxidation layer including mainly $\mathrm{ZrO}_{2}$ and $\mathrm{SiO}_{2}$ [16]. Polishing out the oxidation layer, we can directly observe the surfaces of the ceramics, as shown in Fig. 1(b).

In order to increase the resistance to surface cracking and enhance the rate of surface heat transfer, the surfaces of the specimens were biomimetically made by high temperature plasma etching techniques and chemically corroded by the mixed solution of $\mathrm{HNO}_{3}$ and HF. As a result, 


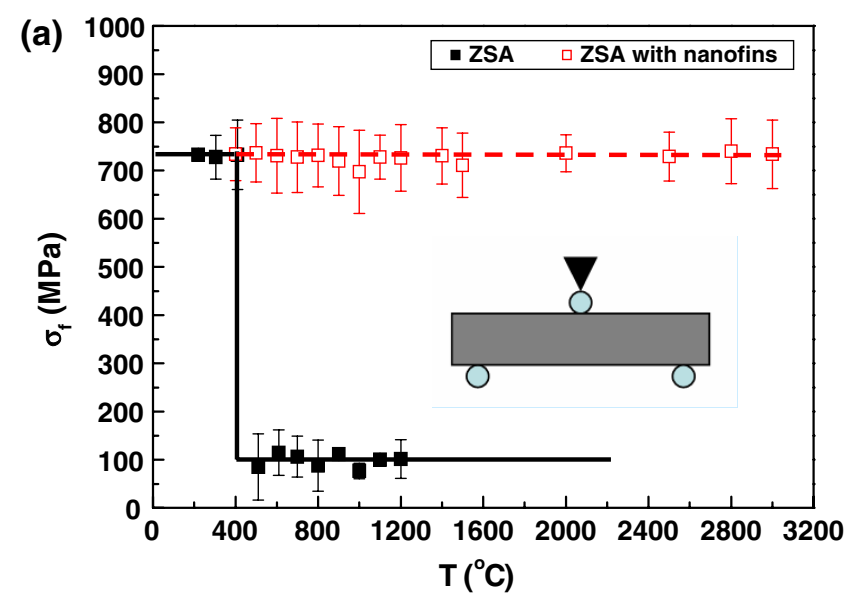

(b)
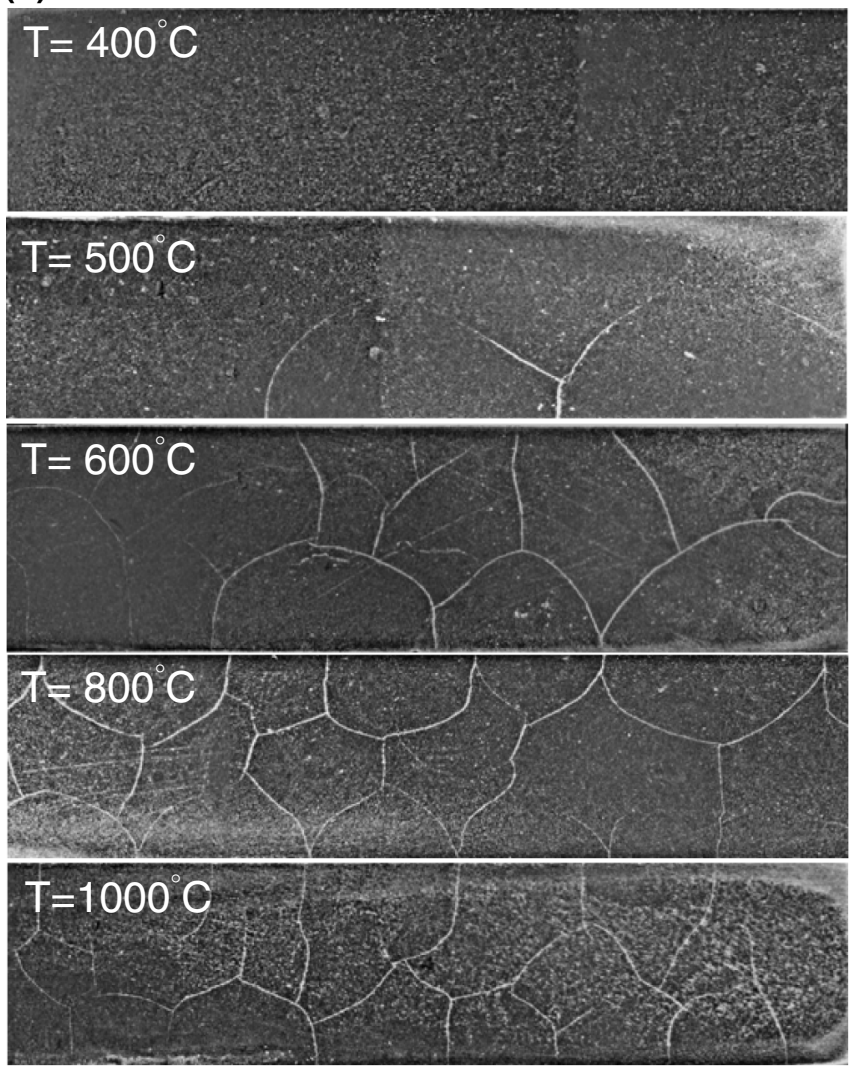

FIG. 1 (color online). (a) Experimental results showing the changes of the flexural strength of the ZSAs without and with nanofins along with the different temperatures of thermal shock. (b) Microscope images showing the surface cracks of the ZSAs without nanofins quenched in room temperature water; the initial temperature of the ZSAs are $400,500,600,800$, and $100{ }^{\circ} \mathrm{C}$, respectively.

surfaces become rough with nanoscale circular rod fins like the surface microstructure of the wing membranes of dragonfly [17], as shown in Fig. 2. These nanofins stand up almost vertically and are randomly distributed on the surfaces of the ceramics. The average diameter, thickness, and numerical density of the nanofins were measured to be $d=81.52 \pm 15.29 \mathrm{~nm}, \quad L=375.18 \pm 32.06 \mathrm{~nm}, \quad$ and
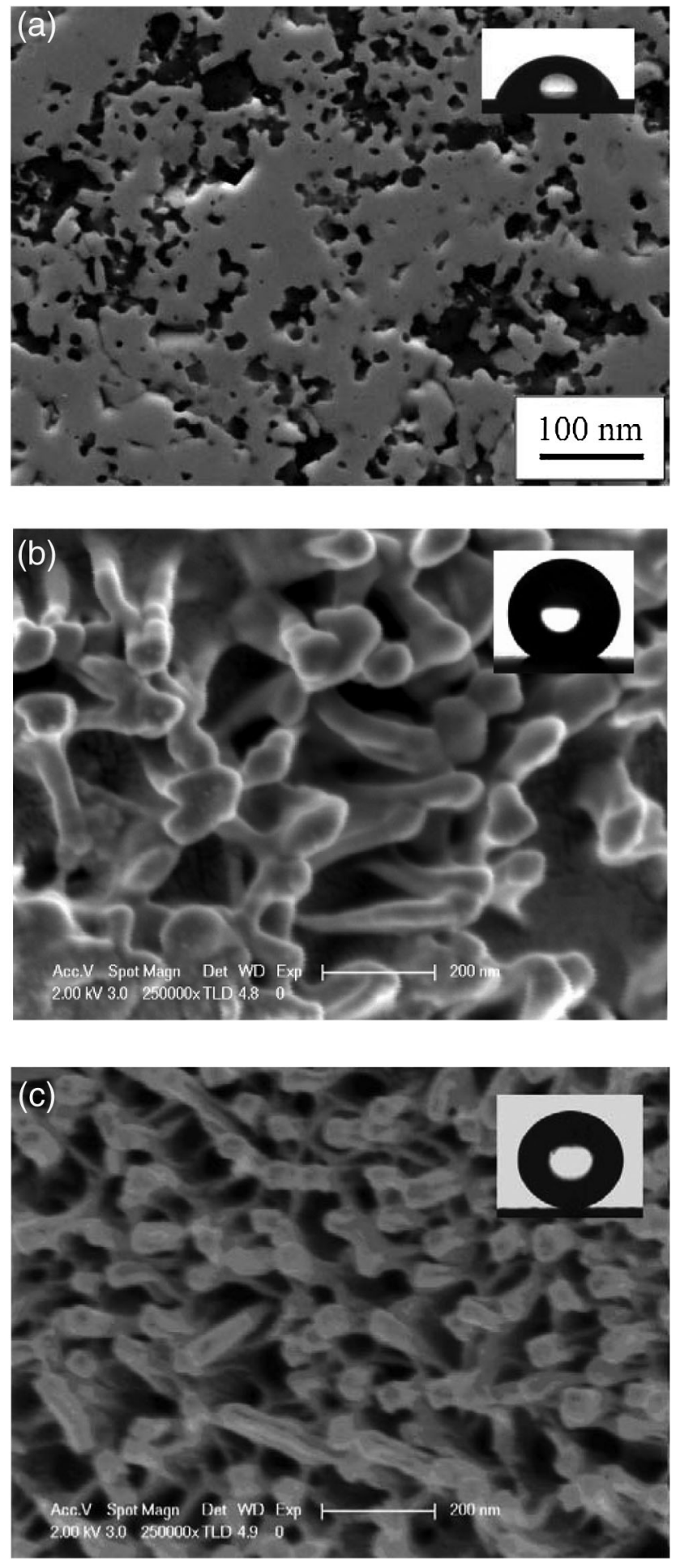

FIG. 2. SEM images showing different surface structures. (a) The surface structure of the ZSA without nanofins, which displays the water contact angles of $70.6 \pm 1.8^{\circ}$ showing in the inset; (b) the surface structure of the ZSA with nanofins, which displays the water contact angles of $121.6 \pm 2.2^{\circ}$ showing in inset; and (c) the surface structure of the dragonfly (Libellula basilinea McLachlan) wing, whose nanofins are mimicked in roughening by the surface of ZSA and displays water contact angles of $174.21 \pm 2.19^{\circ}$ showing in the inset (16). 
$n=71 \pm 12 \mu \mathrm{m}^{-2}$, respectively. The areal fractions of the nanofins and the void space were calculated to be $f_{M}=$ $n \pi d^{2} / 4 \approx 0.37$ and $f_{V}=1-f_{M} \approx 0.63$, respectively, on the rough surface of the ceramic so that the actual surface area was increased about $n \pi d L \approx 724 \%$.

We further tested the flexural strength of the ceramics with the nanofins after thermal shock, and found that the strength was retained up to the melting point, as shown in Fig. 1. This result was an indication that these nanofins effectively enhanced the heat transfer resistance of the interface between the ceramics and the quenching medium so that the thermal gradient and stresses produced on the surface of the ceramics were largely decreased during thermal shock. In particular, no cracks were observed on the tops of the nanofins after thermal shock.

To analyze the physical mechanisms of the experiments above, the structure of the interface between the tested ceramics and the quenching medium was first studied. Obviously, the rough surface made of the nanofins displayed a typical hydrophobic structure [18,19], in a similar way to the dragonfly. Based on the sessile water-drop measurements, the contact angles of water droplets on the surfaces with and without the nanofins were measured to be $\theta_{r}=121.6 \pm 2.2^{\circ}$ and $\theta_{E}=70.6 \pm 1.8^{\circ}$, respectively, as shown in the Fig. 2 inset. Using the CassieBaxter model [20], $\cos \theta_{\mathrm{CB}}=-1+f_{M}\left(\cos \theta_{E}+1\right)$, we readily computed the average water contact angles of the surfaces with the nanofins to be about $120.5^{\circ}$, which was in good agreement with the experimental results. This demonstrated that there was a thin air layer created by the nanofins on the actual surfaces of the ceramics when the ceramics quenched into water, the average thickness of which was approximately equal to that of the nanofins according to the Cassie-Baxter model [18-20], as shown in Fig. 3, inset A. Therefore, the nanofins not only decreased the surface energy of the ceramics but also yielded a strong water-repellent force so as to make water not be able to contact directly the actual surface of the ceramics during quenching [21].

Further, we investigated the effects of the void space created by the nanofins on the heat transfer resistance at the interface between the surface of the ceramic and the quenching medium. When the heated ceramic with the nanofins was quenched into water, the total resistance at the interface can be expressed as $R_{t}=R_{M}+R+R_{W}$, which has contributions from the three regions: the internal region of the ceramic with a characteristic thickness of $L_{M}$, the surface region of the void space with a characteristic thickness of $L$, and the contact region of water with a characteristic thickness of $L_{W}$, as shown in Fig. 3, inset A. From the theory of heat transfer, we obtain $R_{M}=L_{M} / k_{M} \approx 1.4 \times 10^{-5} \mathrm{~m}^{2} \mathrm{~K} \cdot \mathrm{W}^{-1}$, where $k_{M} \approx$ $60 \mathrm{~W} \cdot(\mathrm{m} \cdot \mathrm{K})^{-1}$ is the thermal conductivity of the ceramic [13] and $L_{M} \approx 0.82 \mathrm{~mm}$ is the characteristic dimension of the tested ceramic which is roughly equal to the

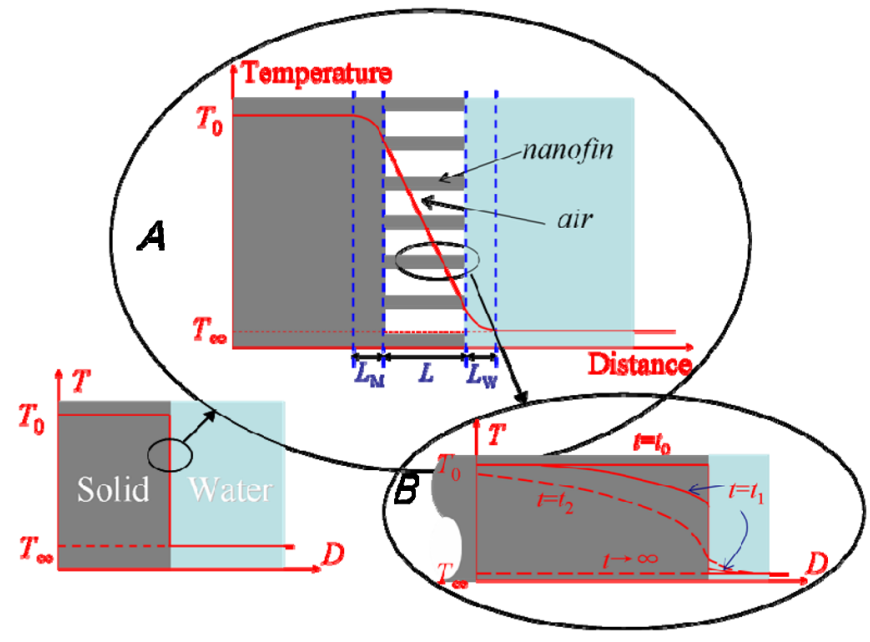

FIG. 3 (color online). Schematic illustrations showing the temperature distribution at the interface between the solid with nanofins and water during quenching. $T_{0}$ and $T_{\infty}$ are the initial temperature of the solid and the temperature of water, respectively. Inset (A) the interfacial structure composed of the solid, the nanofins, the entrapped air, and water, where $L_{M}, L$, and $L_{W}$ stand for the lengths of the regions of internal conduction, the interface, and the surface convection resistance, respectively. Inset (B) the evolution of the temperature profile at the contact interface between the top of a nanofin and water, which is the same as the interface between the surface of the solid without the nanofins and water.

ratio of the volume and the surface area of material, $R_{W}=$ $1 /\left(f_{M} h_{W}\right) \approx 2.7 \times 10^{-4} \mathrm{~m}^{2} \mathrm{~K} \cdot \mathrm{W}^{-1}$, where $h_{W}$ is the forced convection coefficient of water and is taken as on the order of magnitude of $1.0 \times 10^{4} \mathrm{~W} \cdot\left(\mathrm{m}^{2} \cdot \mathrm{K}\right)^{-1}$ (uncertainties of the convection coefficient of \pm 25 percent were quite common in practice [22,23]), and $R \approx$ $f_{M}\left(L / k_{M}\right)+f_{V}\left(1 / h_{A}\right) \approx 0.63 \mathrm{~m}^{2} \mathrm{~K} \cdot \mathrm{W}^{-1}$, where $h_{A}$ is the natural convection coefficient of the entrapped air in the void space created by the nanofins on the surface of the ceramic, which is of the order of magnitude of $1.0 \mathrm{~W}$. $\left(\mathrm{m}^{2} \cdot \mathrm{K}\right)^{-1}[22,23]$. Therefore, the total resistance at the interface was obtained as $R_{t} \approx 0.63 \mathrm{~m}^{2} \mathrm{~K} \cdot \mathrm{W}^{-1}$, which is an indication that the total resistance was predominately determined by that of the entrapped air in the void space created by the nanofins. Moreover, the radiation resistance in the void space was not considered here because it is very small in comparison with the convection resistance yielded by the entrapped air in the void space.

In particular, we compared the resistance of the ceramic without the nanofins with that of the ceramic with the nanofins. When the heated ceramic without the nanofins was quenched into water, the total resistance at the interface was $r_{t}=L_{M} / k_{M}+1 / h_{W} \approx 1.14 \times 10^{-4} \mathrm{~m}^{2} \mathrm{~K} / \mathrm{W}$. Therefore, the resistance of the ceramic with the nanofins was $R_{t} / r_{t} \approx 5.53 \times 10^{3}$ times greater than that of the ceramic without the nanofins. Additionally, the Biot number of the ceramic with the nanofins is $R_{M} /\left(R+R_{W}\right) \approx$ $2.2 \times 10^{-5}$, which is much less than 0.1 and implies that 
the temperature of the interior of the ceramic during quenching was uniformly descendent [22,23], while a steep temperature difference occurred at the interface composed of the nanofins, as shown in Fig. 3, inset A. Therefore, the strong thermal gradient and stresses produced by the steep temperature difference only acted on the nanofins rather than the actual surface of the tested ceramic. Because each of the nanofins was able to expand freely along the direction of their length, there were no strong thermal stresses produced in the interior of the nanofins, i.e, the nanofins did not fail during thermal shock. In particular, there are the stress concentrations in the immediate vicinity of nanofins during quenching, but the level of the stress concentrations in comparison with the intrinsic strength of the material is too low to result in the nanofins and material itself failure.

In conclusion, the main role of nanofins is to confine all the temperature gradient within the air-nanofin layer so that the temperature profile remains almost flat within the ceramics during the whole quenching. And since thermal stresses are proportional to temperature gradient rather than temperature, they remain small within ceramics during the whole quenching. Therefore, the high resistance produced by the entrapped air in the nanofins is such that the ceramics never exhibited the catastrophic reduction of strength and failure by thermal shock until their melting points. The method presented here will be extremely helpful to enable existing ceramics to break through the limit of thermal shock in most thermostructural applications.

We are very grateful to Professor Marc. A. Meyers of UCSD for patiently and helpfully modifying the manuscript. This work was supported by the National Natural Science Foundation of China (Grants No. 10672164, No. 90716004, No. 10732050, No. 10721202) and CAS Innovation Program (Grant No. KJCX2-YW-M04).
*To whom correspondence should be addressed. songf@lnm.imech.ac.cn

[1] D. J. Green, An Introduction to the Mechanical Properties of Ceramics (Cambridge University Press, Cambridge, U.K., 1998).

[2] R.W. Davidge, Mechanical Behaviour of Ceramics (Cambridge University Press, Cambridge, U.K., 1979).

[3] W. G. Fahrenholtz, G. E. Hilmas, I. G. Talmy, and J. A. Zaykoski, J. Am. Ceram. Soc. 90, 1347 (2007).

[4] W. D. Kingery, J. Am. Ceram. Soc. 38, 3 (1955).

[5] D. P. H. Hasselman, J. Am. Ceram. Soc. 46, 535 (1963).

[6] D. P. H. Hasselman, J. Am. Ceram. Soc. 52, 600 (1969).

[7] T. K. Gupta, J. Am. Ceram. Soc. 55, 249 (1972).

[8] H. A. Bahr, U. Bahr, and A. Petzold, Europhys. Lett. 19, 485 (1992).

[9] T. J. Lu and N. A. Fleck, Acta Mater. 46, 4755 (1998).

[10] P. Pettersson, M. Johnsson, and Z. Shen, J. Eur. Ceram. Soc. 22, 1883 (2002).

[11] J. Absi and J. C. Glandus, J. Eur. Ceram. Soc. 24, 2835 (2004).

[12] J. C. Han, Acta Mater. 55, 3573 (2007).

[13] A. L. Chamberlain, W. G. Fahranholtz, G. E. Hilmas, and D. T. Ellerby, J. Am. Ceram. Soc. 87, 1170 (2004).

[14] S. R. Levive et al., J. Eur. Ceram. Soc. 22, 2757 (2002).

[15] S. H. Meng, G. Q. Liu, Y. Guo, X. H. Xu, and F. Song, Mater. Des. 30, 2108 (2009).

[16] J. Liang et al., Scr. Mater. 61, 656 (2009).

[17] F. Song, K. W. Xiao, K. Bai, and Y. L. Bai, Mater. Sci. Eng. A 457, 254 (2007).

[18] R. Blossey, Nature Mater. 2, 301 (2003).

[19] A. Lafuma and D. Quere, Nature Mater. 2, 457 (2003).

[20] A. B. D. Cassie and S. Baxter, Trans. Faraday Soc. 40, 546 (1944).

[21] X. F. Guo and L. Jiang, Nature (London) 432, 36 (2004).

[22] J.P. Holman, Heat Transfer (McGraw-Hill Education (Asia) Co. China Machine Press, Beijing, 2002).

[23] F. P. Incropera, D. P. DeWitt, T.L. Bergman, and A.S. Lavine, Fundamentals of Heat and Mass Transfer (John Wiley \& Sons, Inc., New York, 2007), 6th ed. 